\title{
Avalanche statistics from data with low time resolution
}

\author{
Michael LeBlanc, ${ }^{1}$ Aya Nawano, ${ }^{1}$ Wendelin J. Wright,,${ }^{2,3}$ Xiaojun Gu, ${ }^{2}$ J. T. Uhl, ${ }^{*}$ and Karin A. Dahmen ${ }^{1}$ \\ ${ }^{1}$ Department of Physics and Institute of Condensed Matter Theory, University of Illinois at Urbana-Champaign, 1110 West Green Street, \\ Urbana, Illinois 61801-3080, USA \\ ${ }^{2}$ Department of Mechanical Engineering, One Dent Drive, Bucknell University, Lewisburg, Pennsylvania 17837, USA \\ ${ }^{3}$ Department of Chemical Engineering, One Dent Drive, Bucknell University, Lewisburg, Pennsylvania 17837, USA
}

(Received 31 May 2016; published 22 November 2016)

\begin{abstract}
Extracting avalanche distributions from experimental microplasticity data can be hampered by limited time resolution. We compute the effects of low time resolution on avalanche size distributions and give quantitative criteria for diagnosing and circumventing problems associated with low time resolution. We show that traditional analysis of data obtained at low acquisition rates can lead to avalanche size distributions with incorrect power-law exponents or no power-law scaling at all. Furthermore, we demonstrate that it can lead to apparent data collapses with incorrect power-law and cutoff exponents. We propose new methods to analyze low-resolution stress-time series that can recover the size distribution of the underlying avalanches even when the resolution is so low that naive analysis methods give incorrect results. We test these methods on both downsampled simulation data from a simple model and downsampled bulk metallic glass compression data and find the methods recover the correct critical exponents.
\end{abstract}

DOI: 10.1103/PhysRevE.94.052135

\section{INTRODUCTION}

Avalanches are observed in a variety of dynamical systems, including magnetic materials [1,2], charge density waves [3,4], vortices in superconductors [5], earthquakes [6,7], crystal plasticity [7-11], and amorphous plasticity [7,8,12-17]. For these last two cases, deformation occurs through small jumps caused by slipping weak spots in the material. The slips can trigger other weak spots to slip, and this chain reaction is called an avalanche. The often-observed power-law distribution of avalanche sizes is believed to reflect proximity to a nonequilibrium critical point. A simple mean-field model [18] that exhibits tuned critical behavior in the depinning universality class agrees with experimental data on slip avalanches during the slow deformation of crystalline materials [10], amorphous materials [13], and granular materials [7,19], as well as many other systems with yielding behavior [7].

The avalanches that comprise plastic deformation take place on short time scales (typically on the order of milliseconds in bulk metallic glasses (BMGs) and single crystals [20-22]), so experimental time resolution is important. There are two types of avalanche events observed for BMGs. We have previously referred to these events as small and large avalanches [13,23]. The small avalanches show a power-law size distribution and self-similar dynamics and are likely related to incipient shear bands. The large avalanches are less frequent but almost regularly recurring and do not show a power-law size distribution. The large shear bands are clearly related to the propagation of fully formed, system-spanning shear bands $[13,23]$. We focus here on the small events.

In this paper we demonstrate the effect of insufficient resolution on typical avalanche observables and propose methods to diagnose and mitigate problems due to resolution. We use a simple model for demonstration and to make certain calculations explicit, but many of the results are generic. We find that naive analysis of low-resolution data can lead

\footnotetext{
${ }^{*}$ Retired.
}

to avalanche size distributions with incorrect power-law exponents and even no power-law regime at all. We also show that, with the traditional methods, an apparent data collapse with incorrect exponents can be obtained. (By "incorrect" exponents, we mean exponents different from those obtained analyzing high-resolution data. We maintain this usage throughout the paper.) More importantly, we introduce improved analysis methods that circumvent these problems. We then apply these analysis methods to downsampled high-resolution experimental data on compressed bulk metallic glasses and find good agreement with results previously obtained at high resolution [13]. The results of this study resolve an apparent discrepancy between the results from experiments at different time resolutions reported in the literature. We find that in the experimental data from [13], the measured avalanche size distribution changes from a broad power law (at a data acquisition rate of $100 \mathrm{kHz}$ ) to a peaked distribution once the resolution is lowered to around $50 \mathrm{~Hz}$, a minimum required resolution for this experiment [see Fig. 10(b)].

While it is difficult to extract reliable avalanche duration statistics when the sampling rate is lower than the inverse duration of typical avalanches, we show that avalanche size statistics can be measured for much lower resolutions than this. The time scale that determines the required data acquisition rate for measuring the size statistics is the avalanche nucleation rate, which is the number of avalanches triggered per second during the deformation. If the sampling rate is much lower than the nucleation rate, there will be several avalanches per sample and information about the individual avalanche sizes will necessarily be lost. Furthermore, if there are separate avalanches that occur in consecutive sampling intervals, they can be mistaken for one large avalanche. Since the nucleation rate is proportional to the experimental displacement rate and proportional to the system size (see Sec. III below), the required time resolution will be lower for slower driving and smaller systems. Therefore, if an experiment's resolution is too low and increasing the resolution is not feasible, then reducing the displacement rate or the system size can compensate equally well. 

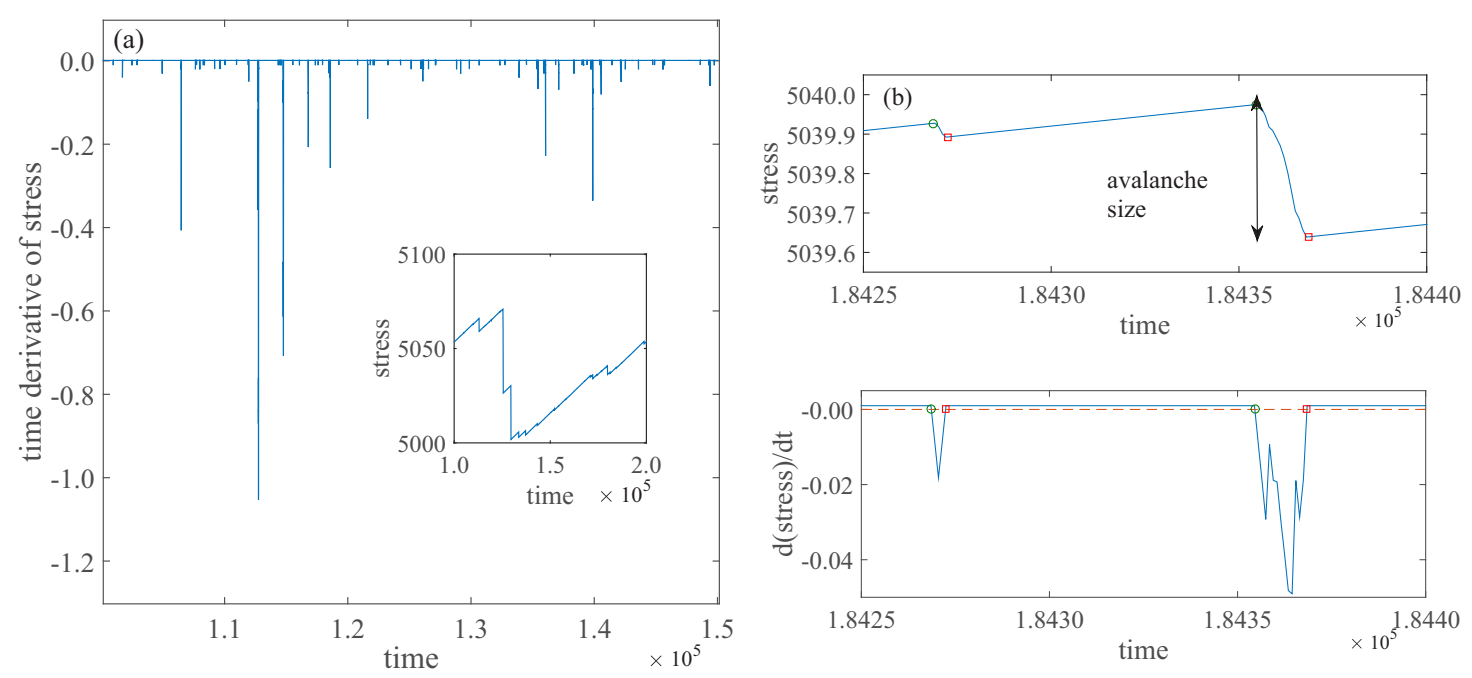

FIG. 1. (a) The time derivative stress vs time series for a typical section of simulation signal from the simple mean-field model (from Ref. [18]). The inset shows the stress vs time curve. (b) A demonstration of what we call the "traditional" avalanche detection procedure. The top figure is a stress-time curve with two avalanches and the bottom is its time derivative. The avalanches are detected when the time derivative drops below a threshold (the dashed line in the bottom figure). The circles and squares indicate the starts and ends of the avalanches, respectively. The avalanche size is the total decrease in stress, as indicated in the top figure.

In this paper, we focus on stress-drop avalanches that occur in experiment at fixed displacement rates. We propose the following:

(1) When the sampling rate is higher than the nucleation rate, but lower than the rate of stress increase between avalanches (in units of the size of a typical stress drop), small stress drops can be missed (see Fig. 3). If the rate $r$ of stress increase between avalanches can be estimated, then instead of defining avalanches as continuous stress drops, one should define them whenever the measured rate of stress increase decreases below $r$. This can be implemented by defining a tilted stress-time curve and measuring stress drops relative to that. The tilted curve is given by $F(t)-r t$, where $r$ is the rate of elastic stress increase [see Eq. (13) and Fig. 3]. On the tilted stress-time curve, there is no background increase in the signal, so small avalanches will still be visible as drops in the tilted signal. Alternatively, this procedure can be implemented by moving the threshold from 0 to $+r$ in the detection method shown in Fig. 1. Under ideal circumstances, avalanche sizes can be recovered with good accuracy using this method (details are given in Sec. VI B). In Sec. VI A, we present a method for determining when a stress-time signal is in this regime. The analysis also reveals a way to extract the avalanche size power-law scaling exponent $\tau$ by downsampling data without actually measuring avalanche sizes [see Eq. (11)].

(2) When the data acquisition rate is slightly lower than the nucleation rate, several avalanches can occur per sampling time. Furthermore, when distinct avalanches happen during successive samples, they will be part of one continuous drop in the low-resolution stress-time signal (see the inset of Fig. 6). For this reason, the stress drops that occur between successive samples will be more representative of the underlying avalanche sizes than the (potentially much larger) consecutive stress drops of the traditional avalanche definition, which potentially involve many time steps (see Fig. 6). Therefore, at these very low resolutions, one should again tilt the signal by subtracting $r t$ and take the stress drops between two successive stress measurements as the best approximation of the avalanche sizes. This will not recover the true size distribution exactly, but the result will be similar to it as long as the data acquisition rate is on the order of the nucleation rate. In Sec. VI C, we give the relevant details and show the expected corrections to the size distribution.

In Sec. VI D, we show that these procedures can be used to obtain a scaling collapse from the low-resolution data with the same critical exponents as one would find at high resolution. We also show that traditional analysis of lowresolution simulation data from the simple mean-field model (of Ref. [18]) at different spring stiffnesses can give a collapse with incorrect exponents (see Fig. 9). In Sec. VII, we compare the results to downsampled experimental BMG compression data and find good agreement with model predictions.

It is important to note that the model exponents and scaling collapses vary according to temporal resolution if a naive analysis of the data is implemented. This result is independent of any particular model. The goal of this paper is not to repeat the study of the validity of the mean-field model that was performed in Ref. [13], but rather to show how the scaling exponents extracted in experiments at much lower data resolution than the exceptionally high resolution used in Ref. [13] can be affected by low temporal resolution. The data analysis methods presented in this paper are meant to aid the analysis of future experiments at low time resolutions. While we use a mean-field model to make certain calculations explicit, we emphasize that the results are applicable beyond mean-field theory, to any system with avalanches, regardless of universality class. 


\section{EXTRACTING AVALANCHE STATISTICS FROM EXPERIMENTAL SIGNALS}

Experimental data on plasticity are generally acquired as one of two types: stress versus time (at constant displacement rate) or displacement versus time (at a constant rate of stress increase). In the first case, an avalanche manifests as a sudden drop in stress and in between avalanches there are "quiet periods" during which the stress increases at a constant rate due to elastic loading. In the second case the avalanches are jumps in the displacement and there is little change in displacement during the quiet periods. As mentioned above, in this paper, we focus on analyzing stress versus time data at a fixed imposed displacement rate, although the results can be adapted in a straightforward fashion to displacement versus time data.

Traditionally, avalanches are detected by taking a derivative of the signal and observing when it drops below a negative threshold. For instance, a stress-drop avalanche begins when the derivative of the stress becomes negative and ends when it returns to being positive (shown in Fig. 1). We call this the traditional (or naive or conventional) method, in contrast to methods we develop in Secs. VI B and VI C that are more appropriate for low-resolution data.

In practice, experimental noise has a large effect on the numerical derivative of a signal, so naive application of this method can be unreliable, especially at high sampling rates. This problem can be addressed by optimally filtering the input data [24,25], by demanding that the stress derivative drops below some negative threshold (rather than a threshold of zero), or by dismissing avalanches below a certain size as indistinguishable from noise. Deciding on the appropriate method requires care because noise cannot only cause spurious avalanches to be detected, but can also cause avalanches to be incorrectly broken into pieces.

Once the avalanche sizes and durations have been measured, estimates of the statistical distributions of these quantities can be constructed. The traditional way to present a distribution estimate is by using a normalized histogram of the sizes and durations as an approximate probability density function (PDF). Histograms can be very effective when data take integer values or when many avalanches have been collected. When the data values span several orders of magnitude (as avalanche sizes and durations often do), logarithmic binning can be effective for reducing noise in the tail of the distribution; however, any histogramming procedure introduces a new parameter (binning scale) and also, more crucially, averages out information from the data.

For this reason, we prefer to use complementary cumulative distribution functions (CCDFs), even though their visual interpretation is not always as straightforward as it is for PDFs. The CCDF for a random variable $X$ is defined as $C(x)=P(X>x)$. It can be estimated from the data as $\hat{C}(x)=$ \{fraction of samples with value greater than $x$ \}. Furthermore, for independent samples, no information is lost: Each "step" in the CCDF estimate is at the location of a data point, so the entire data set can be recovered (except information about the time order of samples, which is irrelevant if they are independent). Key predictions of avalanche models about scaling forms of size and duration PDFs can be cast equally well in terms of the CCDF $[10,13]$.

\section{MODEL}

To analyze the generic effects of low time resolution on avalanche statistics, we use a simple mean field model [18] with an array of $N$ cells that are elastically coupled to one another as well as to a driving spring that moves at a constant rate. The stress $\tau_{i}$ on cell $i$ is given by

$$
\tau_{i}=\frac{J}{N-1} \sum_{j=1}^{N}\left(u_{j}-u_{i}\right)+K\left(v_{d} t-u_{i}\right),
$$

where $u_{i}$ is the position of the $i$ th cell, $t$ is the time, $J$ is the mean field coupling between the cells, and $K$ is the spring constant of the loading spring connecting a site to the sample boundary that moves at speed $v_{d}$. For a system with linear size $L$, the system's boundary is a distance on the order of $L$ away from the cells, which leads to a loading spring stiffness $K \sim 1 / L$ [26]. This means that for a planar shear band with $N$ cells, we have $K \sim 1 / \sqrt{N}$, and therefore large systems have a naturally small value of $K$.

A cell slips forward by a characteristic amount $\delta u$ when its stress surpasses a failure threshold $\tau_{f}$. This causes a local reduction in stress of amount $\delta \tau_{i}=\delta u(J+K)$ for cell $i$, while every other cell $j \neq i$ sees a stress increase of $\Delta \tau_{j}=\frac{J}{N-1} \delta u$. The increase in the other cells' stresses can cause them to slip in turn. This chain reaction is called an avalanche. The avalanche size is defined to be the amount the total stress $F=\sum_{i} \tau_{i}$ drops during the avalanche.

Assuming that $v_{d}$ is so small that the effect of driving can be neglected during the course of an avalanche, the system has one tuning parameter $k \equiv K / J$ that governs the sizes of avalanches. When a cell fails, it relieves $K \delta u$ stress from the boundary spring. If this is comparable to the amount $J \delta u$ that it redistributes to the other cells, it will be impossible to have large avalanches in the steady state. In the thermodynamic limit $N \rightarrow \infty$, the largest avalanches in the scaling regime have size $S_{a} \sim k^{-2}$ and duration $T_{a} \sim k^{-1}$ and their probability density functions (PDFs) follow the scaling forms [18]

$$
\begin{gathered}
P(S) \sim S^{-3 / 2} \mathcal{F}\left(k^{2} S\right), \\
P(T) \sim T^{-2} \mathcal{G}(k T) .
\end{gathered}
$$

Summing Eq. (1), the total stress on the system is $F=$ $N K\left(v_{d} t-\bar{u}\right)$, where $\bar{u}$ is the average displacement of the cells. The instantaneous stress-drop rate is

$$
-\frac{\delta F}{\delta t}=-N K v_{d}+K n \frac{\delta u}{\delta t}=N K\left(v-v_{d}\right),
$$

where $n$ is the number of cells that fail, $\delta u$ is the characteristic distance a cell slips when it fails, $\delta t$ is the time step, and $v=$ $\frac{n}{N} \frac{\delta u}{\delta t}$ is the instantaneous center-of-mass velocity of the system. Thus, the stress-drop rate is proportional to the difference between the instantaneous velocity and the speed of the sample boundary. So if we measure the size of an avalanche as the total stress drop, it is proportional to $\Delta u-v_{d} T$, where $\Delta u=n \delta u$ is the displacement jump and $T$ is the avalanche duration. The disproportionality $v_{d} T$ between stress drops and displacement jumps becomes unimportant in the quasistatic limit $v_{d} \rightarrow 0$ for any finite avalanche duration $T$. 


\section{TIME SCALES}

The two most important time scales in the problem are the maximum avalanche duration $T_{a}$ and the average interevent time $T_{i}$. The smallest relevant physical time scale is the microscopic scale $\delta t$ set by the rate of slip activations in the material. It is the reference time scale for the problem and serves as the simulation time step in the model.

In the limit of quasistatic driving, the maximum avalanche duration $T_{a}$ scales with distance to criticality $k \equiv K / J$ as $T_{a} \sim \delta t k^{-v z}$, where $v=1$ and $z=1$ for mean-field depinning with long-ranged forces [2]. The other time scales pertaining to the avalanche durations also diverge as $k \rightarrow 0$, but not necessarily with the same exponent. For instance, the average avalanche duration scales as $\langle T\rangle \sim k^{-v z(2-\alpha)}$, where $\alpha$ is the power-law exponent for the duration distribution. In mean field theory (MFT), $\alpha=2$ and $\langle T\rangle \sim \ln \left(k^{-1}\right)$ [18,27].

The interevent times are controlled by the displacement rate. The average interevent time is $T_{i}=\delta f /\left(K v_{d}\right)$, where $\delta f$ is the average amount of added stress required to nucleate an avalanche after the previous one ends. In the cellular model, we have $\delta f \sim \delta \tau / N$, where $\delta \tau$ is the amount a cell's stress is reduced when it slips (as defined in Sec. III), so $T_{i}=\delta \tau /\left(N K v_{d}\right)$.

The ratio of the average interevent time and the maximum avalanche duration is an important quantity. If the interevent times are much larger than the avalanche durations, then there are no temporal overlaps between avalanches and the analysis is simplified. This condition can be obtained by driving the system at a sufficiently slow rate. Demanding that the average interevent time $T_{i}$ is much longer than the maximum avalanche duration $T_{a}=\delta t / k^{v z}=\delta t(J / K)^{v z}$ (with $\nu z=1$ in MFT) gives the condition

$$
v_{d} \ll \frac{\delta \tau}{J N \delta t} \approx \frac{\delta u}{N \delta t},
$$

where $\delta u \approx \delta \tau / J$ is the amount a cell slips when it is triggered, as defined in Sec. III. We will assume that this condition holds throughout this paper, so that avalanches are well separated in time.

\section{AVALANCHE DURATION DISTRIBUTIONS}

If an avalanche has shorter duration than the sampling time $t_{s}$, it is impossible to measure its duration. Therefore, we can only expect our measured duration distributions to be accurate when the sampling time is much less than the maximum avalanche duration $T_{a}$. Even avalanche durations that are on the order of the sampling time will be measured incorrectly due to quantization, so only durations that are much longer than the sampling time will be measured accurately.

Furthermore, duration distributions generally scale over fewer orders of magnitude than the size distribution (since, for example, in MFT, $T \sim S^{\sigma v z}$, where $\sigma v z=1 / 2$ ). Therefore, it is unlikely that one will obtain a reliable picture of the scaling regime for the duration distribution except at sufficiently high time resolution. In our simulations we set the duration cutoff to $T_{a} \approx 100$ time steps and this corresponds to an average duration of around $\langle T\rangle \approx 10$. Even when we use $t_{s}=5$ or $t_{s}=2$, we see an apparent deviation in the duration distribution from the power law observed at full resolution (see Fig. 2).

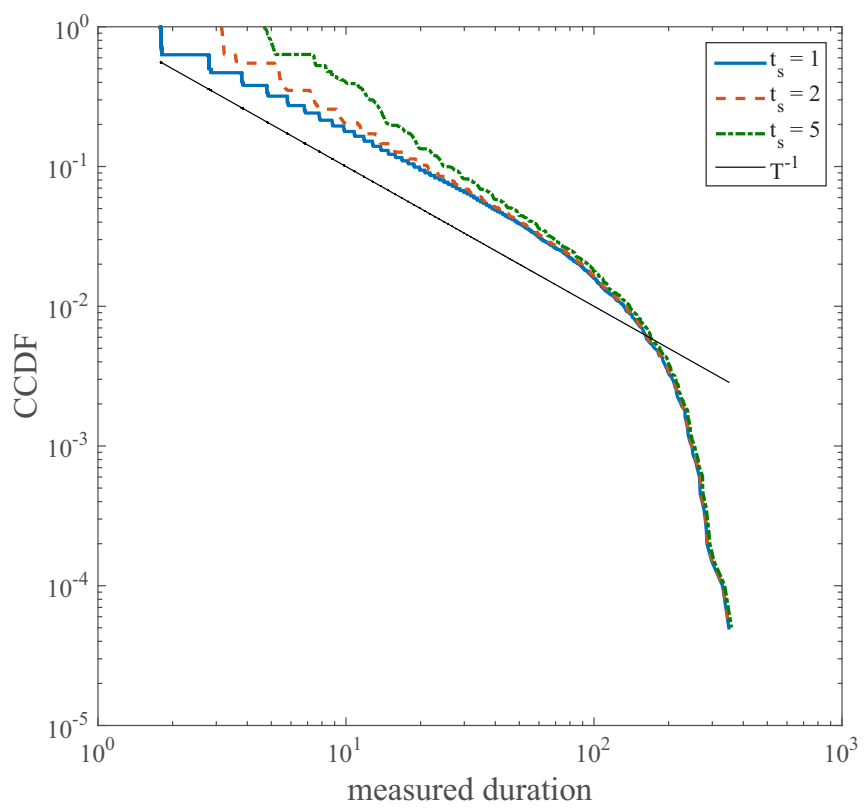

FIG. 2. Duration distributions for several values of the sampling time $t_{s}$. The line represents the MFT prediction $C(T) \sim T^{-1}$ [18]. The apparent power-law changes with sampling time $t_{s}$, even for values of $t_{s}$ much less than the maximum avalanche duration $T_{a} \approx$ 100. The simulation parameters used were number of cells $N=10^{4}$, coupling between cells $J=1$, loading spring stiffness $K=10^{-2}$, and displacement rate $v_{d}=10^{-5}$.

\section{AVALANCHE SIZE DISTRIBUTIONS}

Fortunately, measurements of avalanche sizes are much more robust to low time resolution than durations. This is not because they exhibit more decades of scaling, but rather because stress drops are measurable even if the sampling time is longer than the avalanche duration. Still, there are difficulties with the measurement process as the resolution is decreased, but fortunately some of the most common problems can be mitigated.

We will begin by studying the regime where the sampling time $t_{s}$ is much less than the average interevent time $T_{i}$. The relationship between the sampling time and the maximum avalanche duration $T_{a}$ is of secondary importance when studying avalanche sizes as long as $T_{a} \ll T_{i}$.

\section{A. One avalanche per time step $\left(t_{s} \ll T_{i}\right.$ and $\left.T_{a} \ll T_{i}\right)$}

If we have sampling time $t_{s} \ll T_{i}$, then there will almost always be, at most, one avalanche per time sample (in fact, the avalanche may have a duration of several time samples). In this case, the size distribution of measured avalanches can be computed exactly. The effect of low resolution is that some small stress drops will be overcome by the background elastic increase in stress (see Fig. 3). The elastic stress increase will be $K v_{d} t_{s}$, where $t_{s}$ is the sampling time. The measured stress drop will be $S_{t_{s}}=\min \left(S-K v_{d} t_{s}, 0\right)$ and the avalanches that have size less than

$$
S^{*}=K v_{d} t_{s}
$$



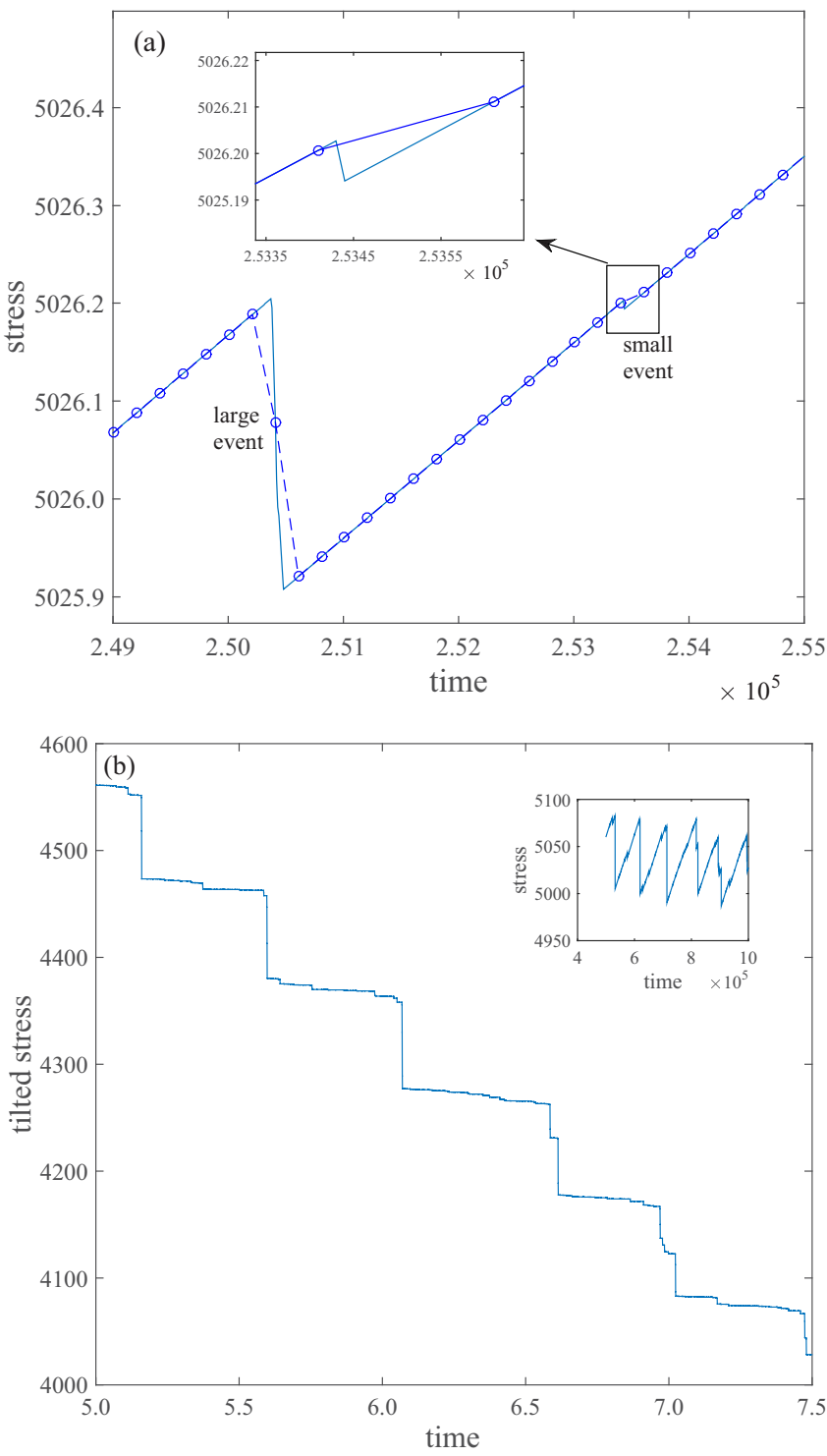

FIG. 3. (a) A portion of a stress-time series from a simulation of the mean-field model (from Ref. [18]). The circles mark every 20th data point and thus correspond to a lower-resolution signal with sampling time $t_{s}=20$ (in units of the simulation time step $\delta t=1$ ). The size of the large event on the left side of the plot is measured fairly accurately by the size of the stress drop in the low-resolution signal. As the inset shows, a small stress drop does not have a corresponding drop in the low resolution signal. (b) A portion of the stress-time curve from the simulation, tilted according to Eq. (13). Subtracting out the elastic stress increase makes the small avalanches visible as drops in the signal. The inset shows the stress-time curve before tilting.

will go undetected by the standard methods. If we assume the avalanches are drawn from the distribution predicted by MFT [27],

$$
P(S)=\frac{\sqrt{S_{m}}}{2 S^{3 / 2}} e^{-k^{2} S / 2},
$$

where $S_{m} \ll k^{-2}$ is the minimum avalanche size, then we will start to notice significant changes in the shape of the size distribution once $S^{*} \gtrsim S_{m}$. The probability that an avalanche

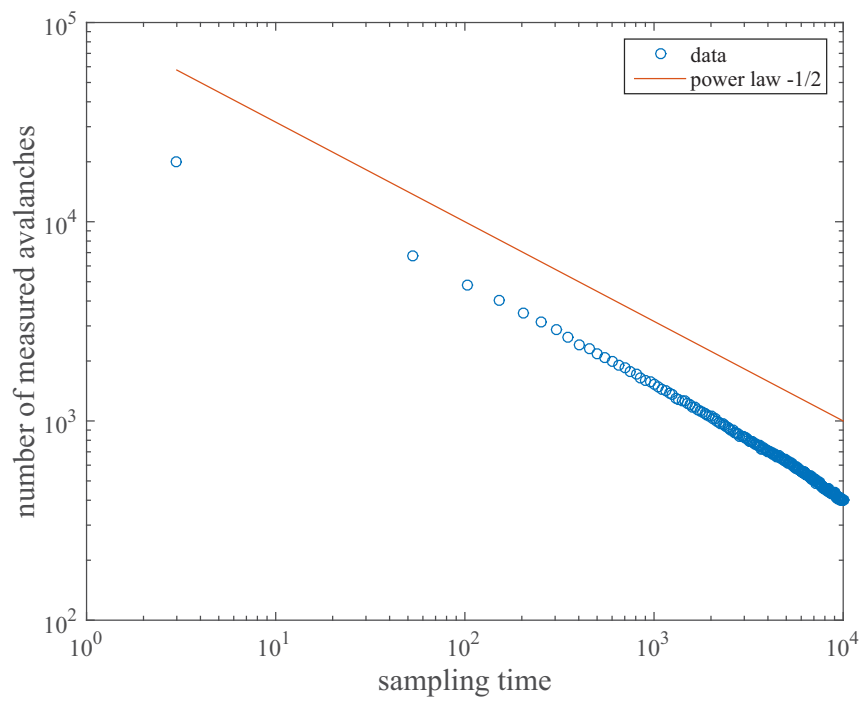

FIG. 4. Number of avalanches collected versus sampling time (in units of the simulation time step $\delta t=1$ ) from a simulation of the mean-field model (from Ref. [18]). The simulation parameters used were number of cells $N=10^{4}$, coupling between cells $J=1$, loading spring stiffness $K=10^{-2}$, and displacement rate $v_{d}=10^{-5}$. The line shows the predicted power law $1-\tau=-1 / 2$ from Eq. (11).

is missed is given by

$$
P\left(S<S^{*}\right)=\int_{S_{m}}^{S^{*}} P(S) d S=\frac{k \sqrt{S_{m}}}{2} \int_{k^{2} S_{m}}^{k^{2} S^{*}} x^{-3 / 2} e^{-x / 2} d x .
$$

If the data acquisition rate is sufficiently high that the largest avalanches are not canceled out by the background stress increase, i.e., if $S^{*} \ll k^{-2}$, then

$$
P\left(S<S^{*}\right) \approx \frac{k \sqrt{S_{m}}}{2} \int_{k^{2} S_{m}}^{k^{2} S^{*}} x^{-3 / 2} d x=1-\sqrt{\frac{S_{m}}{S^{*}}} .
$$

Similarly, for a more general size distribution $P(S) \sim$ $\frac{1}{S^{\tau}} \mathcal{F}\left(S / S_{a}\right)$, where $S_{a}$ is an upper size cutoff and $\mathcal{F}(x)$ is a scaling function that is regular near the origin and decays exponentially at large values, the probability an avalanche will be too small to observe is

$$
P\left(S<S^{*}\right) \approx 1-\left(\frac{S_{m}}{S^{*}}\right)^{\tau-1}
$$

for the case that the largest avalanches can still be detected, i.e., for $S^{*} \ll S_{a}$. This means that as the resolution is lowered, the number of avalanches detected $N_{\text {detected }}$ will go down. The proportion detected $N_{\text {detected }} / N_{\text {total }}$ will be approximately $P\left(S>S^{*}\right)=\left(S_{m} / S^{*}\right)^{\tau-1}$. Since $S^{*} \sim t_{s}$, it follows that

$$
N_{\text {detected }} \sim\left(t_{s}\right)^{-(\tau-1)} .
$$

Interestingly, Eq. (11) gives us a way to estimate the correct size exponent $\tau$ without measuring the size of an avalanche. One can simply downsample the data to lower resolutions (that are still high enough so that large avalanches are detected) and then count the number of avalanches measured at each resolution (see Figs. 4 and 11). Equation (11) also gives a potential way to diagnose whether avalanches are being lost 


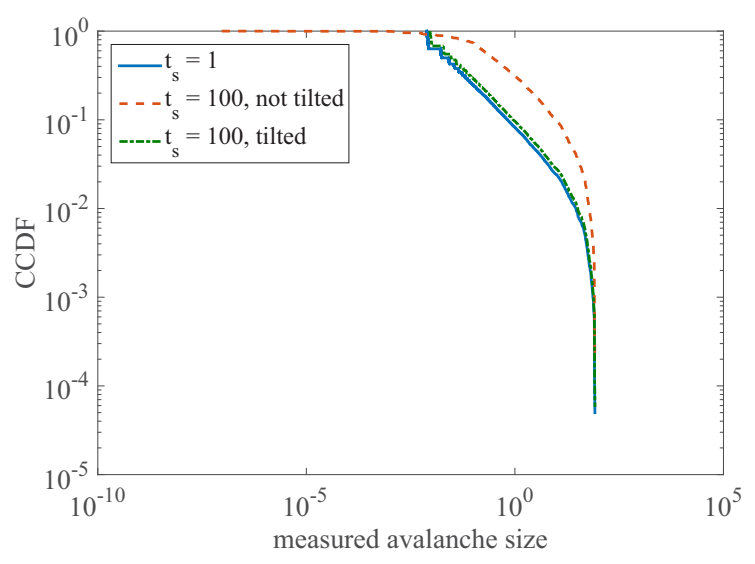

FIG. 5. Measured avalanche size CCDFs at full resolution $\left(t_{s}=\right.$ 1) and at $t_{s}=100$ (about one-tenth of the average interevent time $T_{i}$ ) for both the untilted and the tilted stress-time series. The tilted data give a CCDF that is very close to the one obtained at full resolution, whereas the untilted signal gives a distribution that appears rounded and the power law is difficult to identify by eye. The simulation parameters used were number of cells $N=10^{4}$, coupling between cells $J=1$, loading spring stiffness $K=10^{-2}$, and displacement rate $v_{d}=10^{-5}$.

due to low resolution or high strain rate. If one downsamples the stress-time curve and finds the number of avalanches decays initially like $t_{s}^{\tau-1}$, then avalanches are already being lost due to resolution. If the number of avalanches is constant at first, and then begins to decay, then the resolution is high enough that this effect is not important.

Provided that $S^{*} \ll S_{a}$, the avalanches that are above the detection threshold will come from the truncated distribution $P^{*}(S)=A \frac{\left(S^{*}\right)^{\tau-1}}{S^{\tau}} F\left(S / S_{a}\right)$ for $S>S^{*}$, where $A$ is a numerical constant approximately equal to $\tau-1$ by normalization. Additionally, the observed size of the avalanche is less than its original size by $S^{*}$. So the observed distribution will be shifted,

$$
P_{\mathrm{obs}}(S)=P^{*}\left(S+S^{*}\right)=A \frac{\left(S^{*}\right)^{\tau-1}}{\left(S+S^{*}\right)^{\tau}} F\left[\left(S+S^{*}\right) / S_{a}\right],
$$

for $S>0$. For $S \gg S^{*}$ this looks like the usual power-law decay with a cutoff, but the distribution flattens near the origin. For moderate values of $S^{*}$ the distribution appears rounded and the power law is difficult to identify by eye (see the dashed curve in Fig. 5).

\section{B. Recovering small avalanches for $t_{s} \ll T_{i}$ and $T_{a} \ll T_{i}$}

If the rate of elastic stress increase (i.e., in the absence of avalanches) is known, then deviations from this trend will be approximately the sum of the avalanche sizes during the sampling interval. There are two potential methods for estimating the rate of elastic stress increase. If the stress signal has an elastic portion, its slope can serve as an estimate. Alternatively, as long as the sampling rate is not too low compared to the avalanche nucleation rate, there will be some time intervals where very little plastic deformation occurs that can be used for an estimate.
In the model, the stress increase rate is given by $r=K N v_{d}$. If we estimate $r$, we can tilt the stress signal $F(t)$ to

$$
F(t) \rightarrow F(t)-r t
$$

and then perform the analysis (shown in Fig. 1) on the tilted signal [see Fig. 3(b)]. The complication of small avalanches being covered up by the stress increase during elastic loading is then removed. This procedure can also be understood as moving the detection threshold from 0 to $+r$ in the numerical derivative (see Fig. 1).

For the model signal, we can nearly recover the exact avalanche distribution for $t_{s} \approx T_{i} / 10 \approx T_{a}$. This is possible because we are at a high-enough resolution that with high probability there is at most one avalanche per sampling interval, and therefore no information is lost. Whether the condition $t_{s} \ll T_{i}$ is achieved experimentally depends both on the strain rate and the time resolution. If time resolution is too low, this regime can still be reached by lowering the strain rate.

In the next section, we will discuss the situation where $t_{s} \approx T_{i}$, so that there can be several avalanches per sampling interval.

\section{Multiple avalanches per time step $\left(t_{s} \gtrsim T_{i}\right.$ and $\left.T_{i} \gg T_{a}\right)$}

When the avalanche nucleation rate is on the order of the sampling rate, we must account for the possibility of many avalanches occurring during a time sample. If we attempt to analyze the signal with the method of Fig. 1, two effects become important. First, the stress drop during a sampling time may be from multiple avalanches, and there is no way to know the sizes of the individual avalanches, only their sum. Second, if there are stress drops in consecutive time intervals, they will be all merged into a single avalanche (see Fig. 6). Therefore, the apparent avalanche will only stop once there is a time interval without any stress drop.

If the avalanches are nucleated in a Poisson process, the probability that there are no avalanches during a sampling time interval $t_{s}$ is $p_{0}=e^{-t_{s} / T_{i}}$, where $T_{i}$ is the mean interevent time between avalanches. The measured avalanche durations will be quantized in terms of the sample time, i.e., $T=m t_{s}$, where $m$ is the number of consecutive sample times during which the stress drops. Since the measured avalanche continues until there is a sampling time with no physical avalanches, the duration distribution follows the geometric distribution [28],

$$
P\left(T=m t_{s}\right)=\left(1-p_{0}\right)^{m-2} p_{0},
$$

where $p_{0}=e^{-t_{s} / T_{i}}$ is the probability the stress does not decrease during the time step, thereby ending the measured avalanche. [The factor $\left(1-p_{0}\right)$ on the right-hand side of Eq. (14) is raised to the power $m-2$ instead of the usual $m-1$ for the geometric distribution since the minimum avalanche duration is one rather than zero.] From the distribution defined in Eq. (14), we can compute the average measured duration to be

$$
\langle T\rangle=\frac{t_{s}}{p_{0}}+t_{s}=t_{s}\left(1+e^{t_{s} / T_{i}}\right) .
$$

Likewise, the average size of an apparent avalanche will increase exponentially with sampling time. Since the physical avalanches are nucleated in a Poisson process with rate $1 / T_{i}$, 


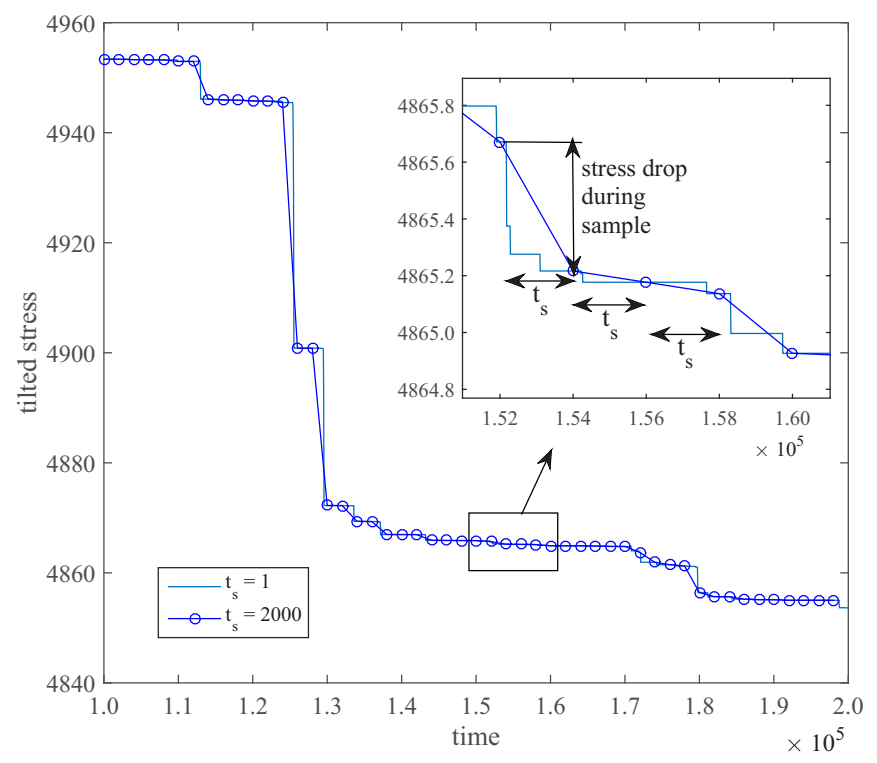

FIG. 6. A portion of a stress-time series from a simulation of the mean-field model (from Ref. [18]), tilted as described in Eq. (13). The circles mark every 2000th data point and thus correspond to a very low-resolution signal with sampling time $t_{s}=2000$. This sampling time is about twice the inverse avalanche nucleation rate, so most increments contain at least one avalanche. As a result, measuring avalanches as successive drops in the tilted stress will give stress drops of very long duration, corresponding to many successive underlying avalanches. However, the stress drops measured between successive sample points will be close in size to the underlying avalanches, although sometimes several avalanches may be merged, as is the case for the first and last time step of size $t_{s}$ of the inset plot. The simulation parameters used were number of cells $N=10^{4}$, coupling between cells $J=1$, loading spring stiffness $K=10^{-2}$, and displacement rate $v_{d}=10^{-5}$.

the number of physical avalanches during a sample will be Poisson distributed with average $t_{s} / T_{i}$. If we let $n$ be the number of physical avalanches that occur during a sample time, $n$ will have the distribution

$$
P(n)=\frac{1}{e^{t_{s} / T_{i}}-1} \frac{\left(t_{s} / T_{i}\right)^{n}}{n !}
$$

for $n \geqslant 1$. We modified the Poisson distribution so to not include $n=0$ because sampling times in which no physical avalanches happen will not show any stress decrease. Taking the average of the distribution,

$$
\langle n\rangle=\sum_{n=1}^{\infty} n P(n)=\frac{t_{s} / T_{i}}{1-e^{-t_{s} / T_{i}}} .
$$

The average measured avalanche size is the product of the average number of sample times per avalanche $\langle T\rangle / t_{s}$, the average number of physical avalanches per sample time $\langle n\rangle$, and the average size of an avalanche, which gives

$$
\langle S\rangle=\frac{\langle T\rangle}{t_{s}}\langle n\rangle\langle S\rangle_{0}=\frac{t_{s}}{T_{i}} \frac{\left(1+e^{t_{s} / T_{i}}\right)}{\left(1-e^{-t_{s} / T_{i}}\right)}\langle S\rangle_{0},
$$

where $\langle S\rangle_{0}$ is the average size of the underlying physical avalanches. In Fig. 7 we show that Eqs. (15) and (18) agree with the simulation of the MFT model.
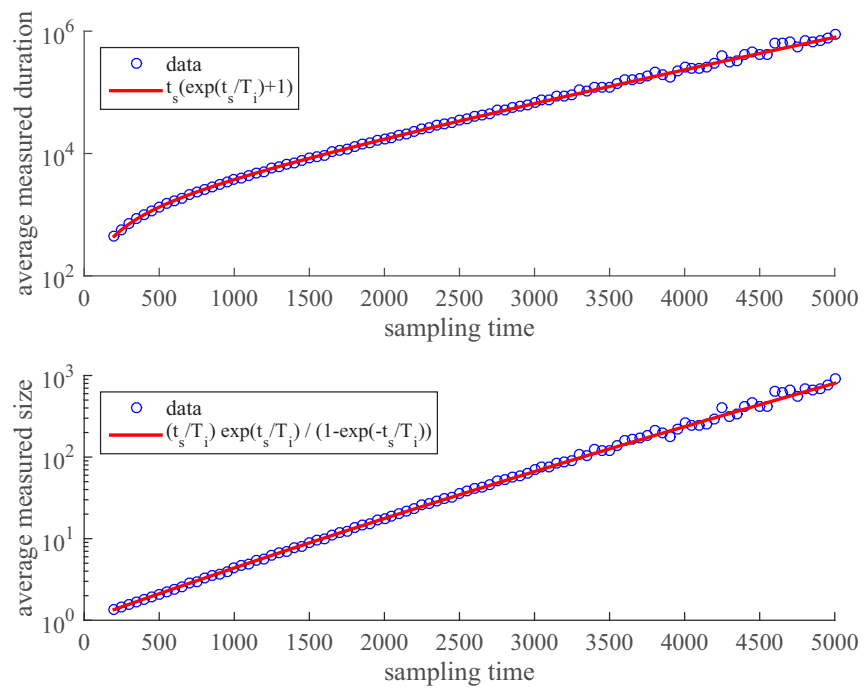

FIG. 7. Mean values of the measured size and duration vs sampling time from the analysis (shown in Fig. 1) of the tilted signal. The curves are the predicted values from Eqs. (15) (top) and (18) (bottom).

In Fig. 8, we see that in this very low-resolution regime, the standard analysis (as shown in Fig. 1) gives something completely different from the underlying avalanche distributions. The principal complication is that consecutive intervals are likely to have stress drops. Therefore, it is more representative to measure the avalanche as the stress drop during a single

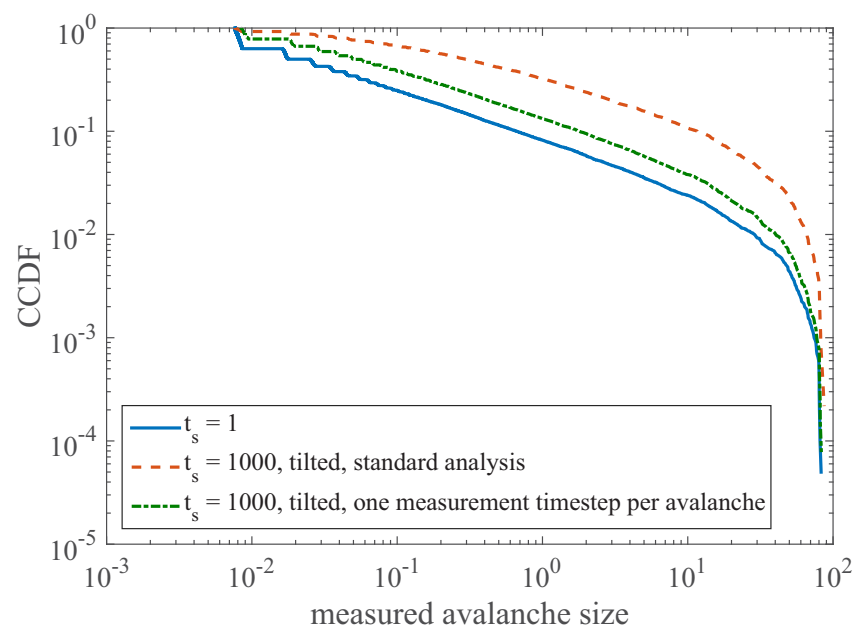

FIG. 8. Avalanche size CCDFs from simulations of the meanfield model (from Ref. [18]) at full resolution and from tilted stress-time curves at low resolution $t_{s}=1000$ (roughly two times the average interevent time $T_{i}$ ) using the analysis described in Fig. 1 and the improved method for very low-resolution data described in Sec. VI C that defines the sample stress drops defined in Eq. (19) (after tilting) as avalanche sizes. The distribution from the improved method is much closer to the full-resolution distribution and the same rough power-law behavior can be seen, but it is still somewhat distorted due to avalanche merging. The simulation parameters used were number of cells $N=10^{4}$, coupling between cells $J=1$, loading spring stiffness $K=10^{-2}$, and displacement rate $v_{d}=10^{-5}$. 

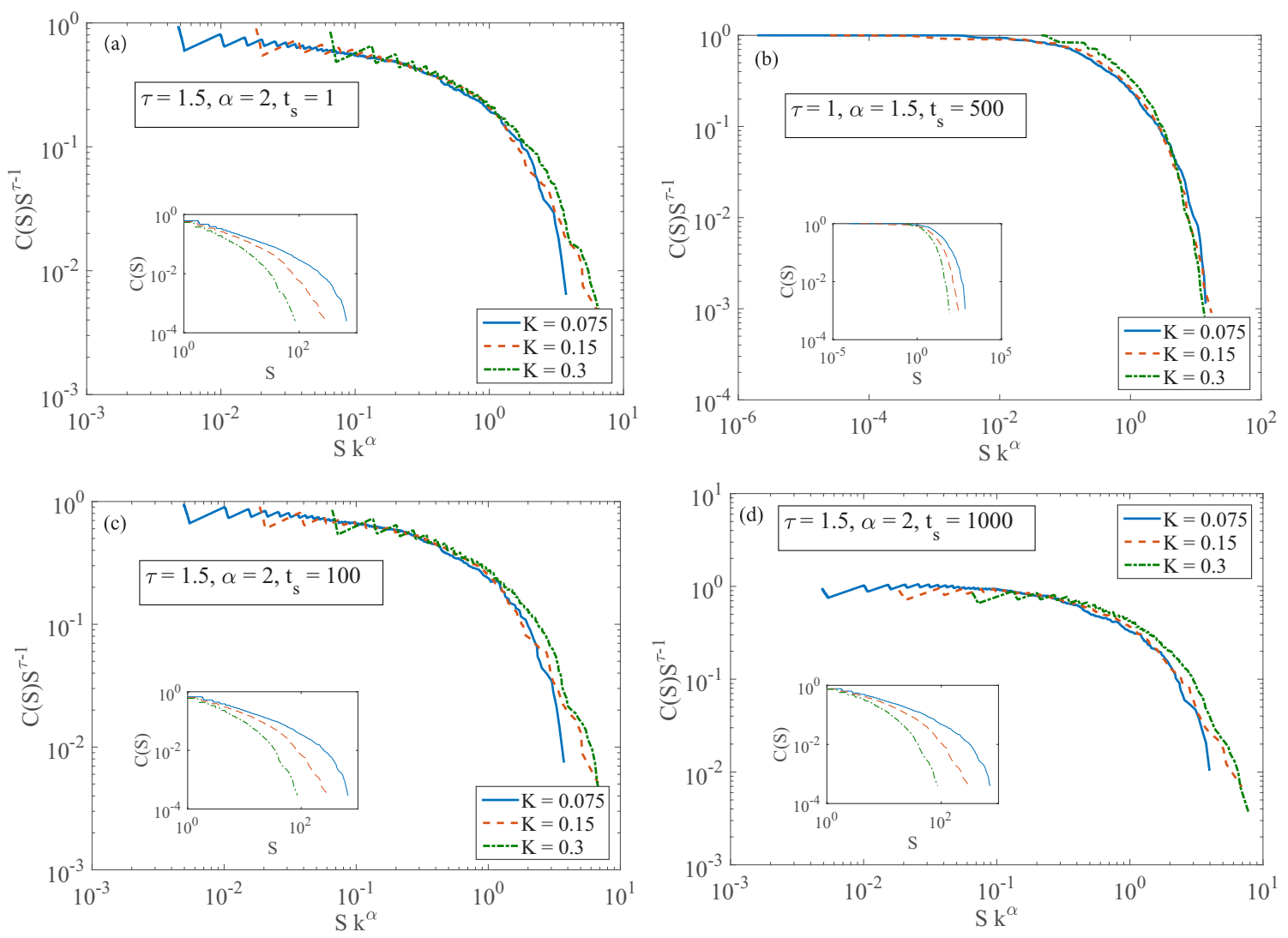

FIG. 9. (a) Data collapse of the size CCDF [Eq. (7)] for different $K$ values from a simulation of the mean-field model (from Ref. [18]) at full resolution. The collapse uses MFT exponents $\tau=3 / 2$ and $\alpha=2$ [18]. (b) An apparent collapse for $t_{s}=500$ using the analysis shown in Fig. 1. Here the collapse yields different exponents $\tau=1$ and $\alpha=1.5$. (c) A collapse with MFT exponents $\tau=3 / 2$ and $\alpha=2$ for $t_{s}=100 \ll T_{i}$, using the the tilted signal described in Sec. VI B. (d) A successful collapse with MFT exponents $\tau=3 / 2$ and $\alpha=2$ for $t_{s}=1000 \approx T_{i}$, using the sample stress drops defined in Eq. (19). Insets are the original distribution before the rescaling.

sampling time. This means that if the stress changes by an amount $\delta F$ during a sampling time, one should record an avalanche of size

$$
S= \begin{cases}-\delta F, & \delta F<0, \\ \text { no avalanche, } & \delta F \geqslant 0\end{cases}
$$

(see the inset of Fig. 6). We will call these the sample stress drops to distinguish them from the stress-drop avalanches that extend over multiple time steps that are measured in the analysis.

One complication with this method is that sometimes there are several avalanches during a time step. We cannot recover the sizes of the individual avalanches, but if we know the nucleation rate, we can predict the distribution of apparent avalanche sizes as follows.

Let $P_{n}(S)$ be the distribution of the sum of $n$ independent avalanches. The distribution of apparent avalanche sizes will be

$$
P(S)=\sum_{n=1}^{\infty} P(n) P_{n}(S)
$$

where $P(n)$ is the distribution of the number of avalanches in a sampling time from Eq. (16) and $P_{n}(S)$ is the distribution of the sum of $n$ independent avalanche sizes. In the Appendix, we show that

$$
P_{n}(S)=\frac{n v_{0}}{\sqrt{2 \pi S^{3}}} e^{-\frac{1}{2 S}\left(k S-n v_{0}\right)^{2}},
$$

where $v_{0}=S_{m} / \delta t$, the ratio of the minimum physical avalanche size to the minimum physical avalanche duration. As long as $\langle n\rangle$, the average number of avalanches per sampling time, is not too big, the distribution of Eq. (20) looks roughly like the distribution $P(S)$ of physical avalanche sizes with a larger short-distance cutoff since the $n=1$ term of Eq. (20) dominates. Provided that $\langle n\rangle\langle S\rangle_{0} \ll S_{a}$, where $\langle S\rangle_{0}$ is the average physical avalanche size and $S_{a}$ is the maximum physical avalanche size, the tail of the distribution is not affected by merging, so a collapse may still look reasonable (see Figs. 8 and 9).

\section{Data collapse at low resolution}

The physical scaling form predicted by the model, $P(S) \sim$ $S^{-\tau} \mathcal{F}\left(k^{\alpha} S\right)$ can be tested by performing a scaling collapse for different $K$ values. This scaling form implies a scaling form,

$$
C(S)=\int_{S}^{\infty} P\left(S^{\prime}\right) d S^{\prime} \sim S^{1-\tau} \overline{\mathcal{F}}\left(k^{\alpha} S\right),
$$

for the CCDF, where $\overline{\mathcal{F}}(x)=\int_{1}^{\infty} z^{-\tau} \mathcal{F}(z x) d z$ [7]. 
We use the loading spring constant $K$ as a tuning parameter for our collapses. There are two complications that we correct for. First, since the definition of the stress drops scales with $K$, the avalanche sizes we use in the collapse are rescaled $S \rightarrow$ $S / K$ before collapsing. We also tune the displacement rate $v_{d}$ so that the interevent time $T_{i} \sim\left(K v_{d}\right)^{-1}$ remains constant. This allows us to tune the spring constant over a wider range without changing the resolution regime (i.e., $t_{s} \ll T_{i}$ or $t_{s} \gtrsim$ $\left.T_{i}\right)$.

In Fig. 9(a) we plot $C(S) S^{\tau-1}$ versus $S K^{\alpha}$, which, according to Eq. (22), should give the plot of the scaling function $\overline{\mathcal{F}}$. We do this for three values of $K$ and confirm that they all lie on the same curve. As we lower the resolution, the distributions become distorted and the scaling form ceases to hold; however, due to regularities in the behavior of the measured size distribution at different resolutions, an apparent data collapse can still be possible. In Fig. 9(b) we show an apparent collapse we obtained for $t_{s}=500$, after performing the naive avalanche analysis of Fig. 1 without tilting the stresstime curve; however, the exponents $\tau=1$ and $\alpha=1.5$ that collapse the data strongly deviate from the MFT values $\tau=1.5$ and $\alpha=2$ that yield a collapse for the full resolution data.

In the lower panels, we show that we can obtain acceptable collapses with the MFT exponents for $t_{s}=100$ and $t_{s}=1000$ when we use the improved analysis methods outlined in Secs. VI B and VI C.

\section{COMPARISON WITH EXPERIMENT}

We compare our theoretical results with experimental data on bulk metallic glasses originally presented in [13]. The data are collected at a very high data acquisition rate of $100 \mathrm{kHz}$ and the avalanche size distribution and average avalanche shapes show good agreement with mean-field theory. See Ref. [13] for details on the experiment and data analysis.

One complication in applying the above analysis to data is the presence of noise. We use Wiener filtering to reduce the noise amplitude (see Ref. [13] for details). Since the analysis uses a numerical derivative to detect stress drops (shown in Fig. 1), even a small amount of noise can have a large effect, and there are still many small events that are a result of noise rather than material response. These "noise avalanches" can be seen as a sharp decrease on the left part of the 100-kHz CCDF in Fig. 10(b). After the sharp decrease, a scaling regime emerges where the size distribution follows the predicted power law $C(S) \sim S^{-1 / 2}$. Avalanches from this region, as well as larger avalanches, are not polluted by noise.

In Fig. 10 we plot the avalanche size CCDFs obtained using the analysis (without tilting; see Fig. 1) at different resolutions from a model simulation and experiment. In both cases, we see that the distribution becomes narrower at lower resolution. In the CCDFs for the experimental data (bottom panel), a pronounced noise regime is visible for the higher resolutions, but disappears at lower resolutions since the noise averages out over long sampling times.

In Fig. 10(b) we see that when the resolution is lowered to around $100 \mathrm{~Hz}$, the power law begins to become shallower and below $50 \mathrm{~Hz}$ the power law begins to disappear entirely. This gives a rough lower bound for resolution at which we expect resolution effects to be important for stress-drop distributions
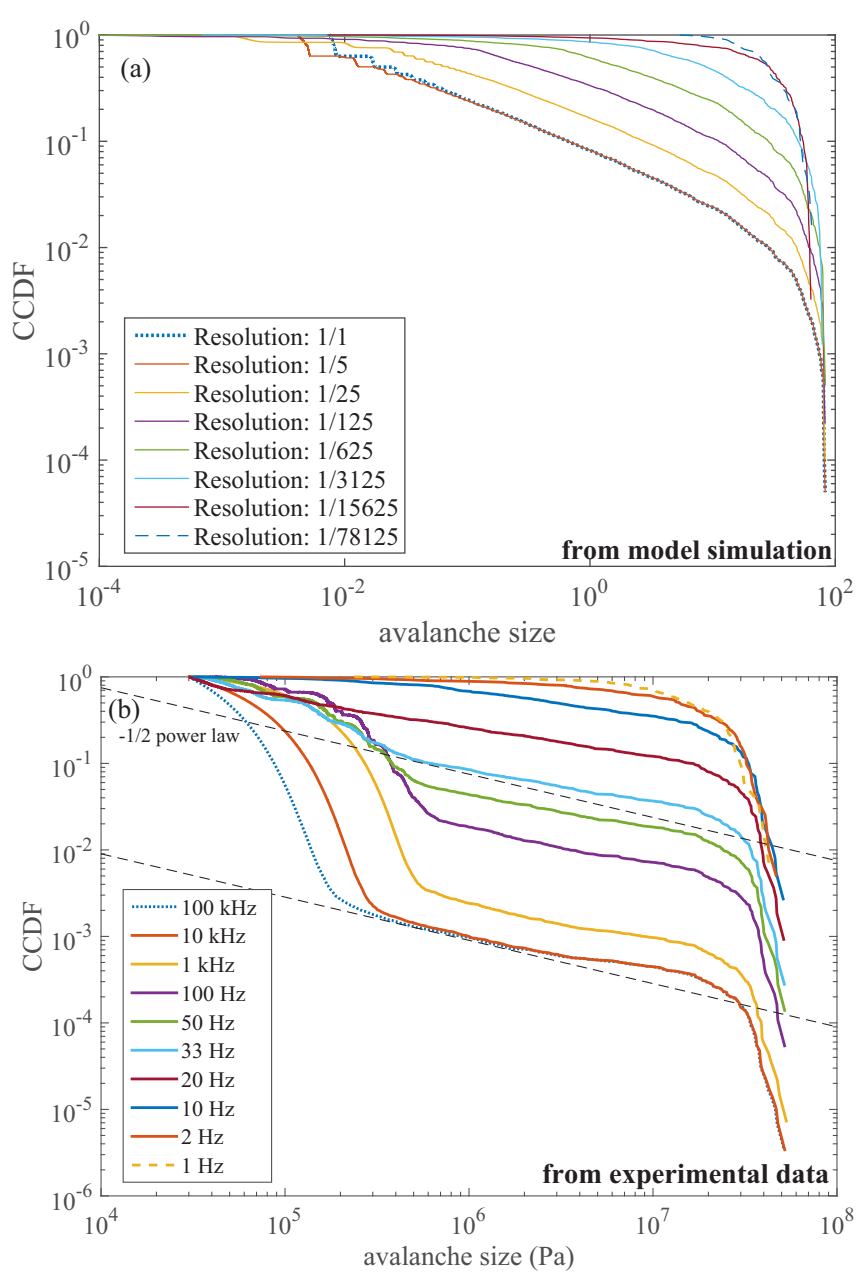

FIG. 10. (a) Avalanche size distributions measured at different resolutions from simulations of the mean-field model (from Ref. [18]) and (b) from BMG experiment (from Ref. [13]). The trend lines are power laws with exponent $1-\tau=-1 / 2$ predicted by MFT. In both cases, the analysis described in Fig. 1 gives avalanche size distributions that become increasingly narrow at low resolution. Thus, low resolution can obscure power-law distributed jumps in the underlying signal. In the experimental data, a pronounced noise regime is visible for the higher resolutions, but is averaged out at lower resolutions.

in this particular system. Since the key time scale is the avalanche nucleation rate [which is proportional to $1 /(N K v)$ ], resolution effects can be avoided by decreasing the system size or decreasing the displacement rate rather than increasing the resolution.

In Fig. 11 we plot the number of avalanches collected versus the sampling time. We see rough agreement with the power law of $1-\tau=-1 / 2$ predicted by Eq. (11) for high resolutions but with a more pronounced deviation for larger sampling times (also see Fig. 4). This is due to the shorter scaling regime in the experimental data and deviations from scaling for large avalanches [13].

Finally, in Fig. 12, we use the method from Sec. VI A to recover the power-law exponent from the experimental data downsampled to $2 \mathrm{~Hz}$, a resolution at which the traditional analysis yields a distribution with no power-law regime. First 


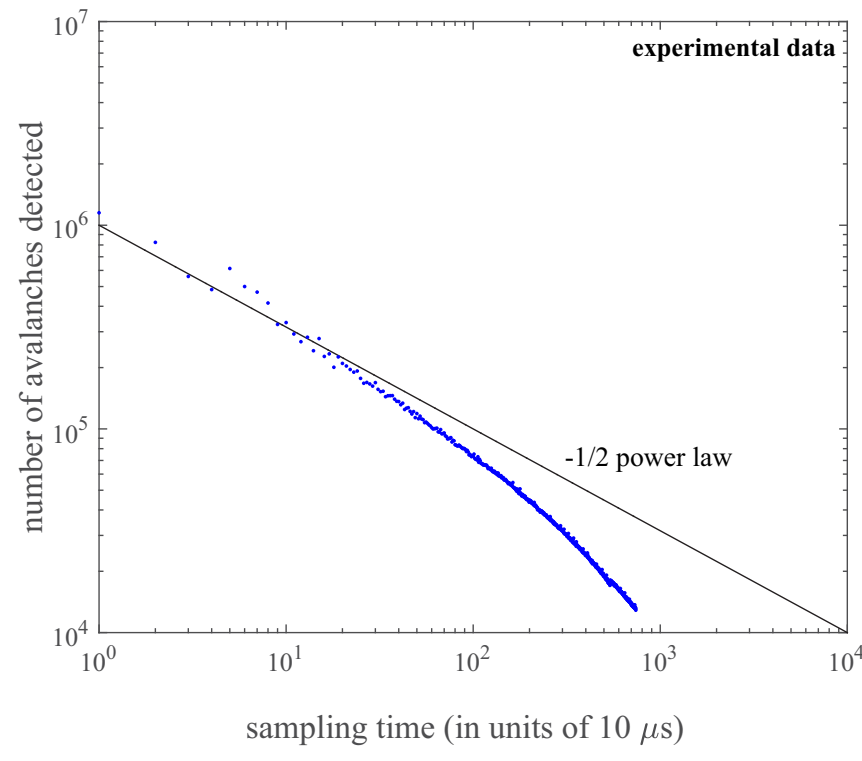

FIG. 11. Number of avalanches collected versus sampling time $t_{s}$ from the experimental data. The line is a power law with exponent $1-\tau=-1 / 2$ from Eq. (11).

the data were cut into sections of monotonically increasing stress, and an appropriate stress increase rate $r$ was determined by a linear fit for each section [see Eq. (13)]. The measured value of $r$ was about $6.5 \pm 0.3 \mathrm{MPa} / \mathrm{s}$ throughout the sample. Then we tilted the lowered-resolution data according to the local values of $r$ and took the distribution of sample stress drops for each sample in which the stress change was negative. The full-resolution power law of $-1 / 2$ is recovered in the resulting CCDF.

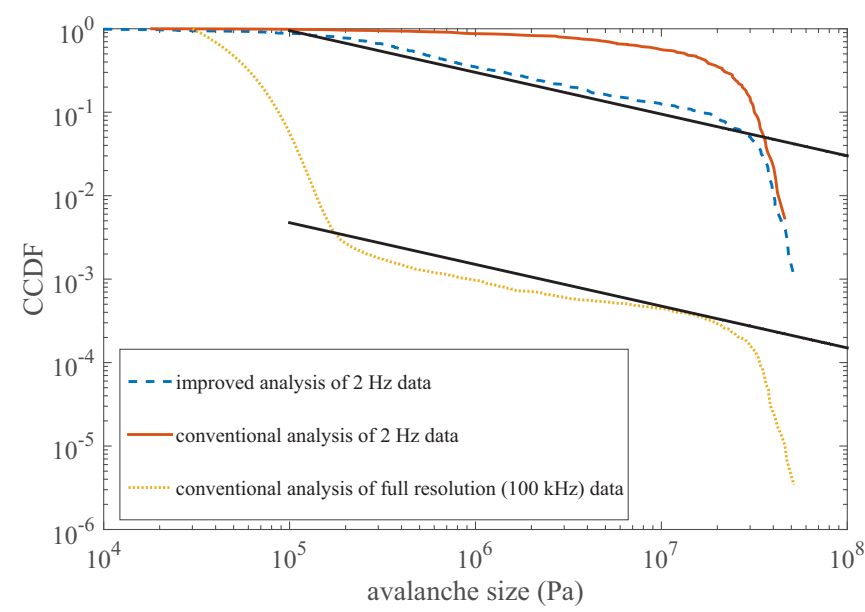

FIG. 12. The bottom curve is the avalanche size CCDF measured using the analysis described in Fig. 1 on the full-resolution $(100-\mathrm{kHz})$ experimental data. The scaling regime follows a power law with exponent $1-\tau=-1 / 2$, in agreement with MFT. The top curve is the CCDF measured with the analysis when the same data are drastically downsampled to $2 \mathrm{~Hz}$. The power law is no longer apparent. The middle curve is the CCDF measured using the improved method outlined in Sec. VI A, where the sample stress drops are analyzed from a tilted signal [see Eq. (19)]. The $-1 / 2$ power law apparent in the $100-\mathrm{kHz}$ data is recovered from the downsampled data.

\section{CONCLUSION}

Lowered resolution affects the measured avalanche distributions in several ways. Avalanche duration distributions are distorted significantly unless the sampling time is well below the average avalanche duration. For larger sampling times (lower resolutions), the apparent power law is steeper than the theoretical power law (see Fig. 2). Avalanche size distributions are much more robust and can be measured even when the sampling time is longer than the duration of the largest avalanches, but there can still be significant rounding of the shape of the size distribution.

For moderately low resolution where the sampling time is still much less than the average time between avalanches, tilting the data before measuring the stress drops makes it possible to recover the underlying avalanche size distribution to a good approximation. For very low resolutions with sampling times on the order of the interevent time, tilting the signal and using the sample stress drops described in Sec. VI C allow the recovery of a size distribution similar to the one that would be obtained at high acquisition rates.

\section{ACKNOWLEDGMENTS}

M.L., K.A.D., and A.N. thank Will McFaul for useful discussions and for help preparing figures. K.A.D., A.N., and M.L. thank MGA, Grants No. NSF CBET 1336634 and No. NSF DMS 1069224 and DOE Grant No. DE-FE0011194 for support. W.J.W. and X.J.G. gratefully acknowledge support from Grant No. NSF DMR 1042734. W.J.W. and K.A.D. thank the Kavli Institute for Theoretical Physics for hospitality and support through the National Science Foundation under Grant No. NSF PHY-1125915.

\section{APPENDIX: DERIVATION OF THE MERGED AVALANCHE DISTRIBUTION}

In the continuum limit, the distribution of avalanche sizes is given by a Brownian first passage time distribution

$$
P(S)=\frac{v_{0}}{\sqrt{2 \pi S^{3}}} e^{-\frac{1}{2 S}\left(k S-v_{0}\right)^{2}},
$$

where $v_{0} \sim S_{m} / \delta t$, with $S_{m}$ the minimum avalanche size and $\delta t$ the minimum avalanche duration [27]. $v_{0}$ may be thought of as the size of the initial "kick" starting an avalanche.

We can then compute the distribution of the sum of two independent avalanche sizes from this distribution. We first Laplace transform, giving

$$
\begin{aligned}
\tilde{P}(\lambda) & =\int_{0}^{\infty} d S e^{-\lambda S} P(S)=\frac{v_{0} e^{k v_{0}}}{\sqrt{2 \pi}} \int_{0}^{\infty} \frac{d S}{S^{3 / 2}} e^{-\left(\lambda+k^{2} / 2\right) S-v_{0}^{2} / 2 S} \\
& =e^{k v_{0}} e^{-\sqrt{2 v_{0}^{2}\left(\lambda+k^{2} / 2\right)}} .
\end{aligned}
$$

By the convolution theorem, the Laplace transform of the distribution of the sum $\Sigma_{n}=\sum_{j=1}^{n} S_{j}$ of $n$ independent draws from $P(S)$ is just $\tilde{P}(\lambda)^{n}$. We can then inverse Laplace transform to get

$$
P\left(\Sigma_{n}\right)=\int_{a-i \infty}^{a+i \infty} \frac{d \lambda}{2 \pi i} e^{\lambda \Sigma_{n}} \tilde{P}(\lambda)^{n},
$$


where $a>-k^{2} / 2$ to avoid the branch cut. The integral can be done by deforming the contour around the branch cut and shifting $\lambda \rightarrow \lambda-k^{2} / 2$, giving

$$
P\left(\Sigma_{n}\right)=\frac{e^{k v_{0}}}{\pi} e^{-k^{2} \Sigma_{n} / 2} \int_{0}^{\infty} d \lambda \sin \left(\sqrt{2 v_{0}^{2} \lambda}\right) e^{-\lambda \Sigma_{n}}=\frac{n v_{0}}{\sqrt{2 \pi\left(\Sigma_{n}\right)^{3}}} e^{-\frac{1}{2 \Sigma_{n}}\left(k \Sigma_{n}-n v_{0}\right)^{2}} .
$$

The sum of $n$ avalanches has the same distribution as a single avalanche with $n$ times as large an initial kick [29].

[1] J. P. Sethna, K. A. Dahmen, and C. R. Myers, Nature (London) 410, 242 (2001).

[2] S. Zapperi, P. Cizeau, G. Durin, and H. E. Stanley, Phys. Rev. B 58, 6353 (1998).

[3] P. A. Lee and T. M. Rice, Phys. Rev. B 19, 3970 (1979).

[4] S. Brazovskii and T. Nattermann, Adv. Phys. 53, 177 (2004).

[5] S. Field, J. Witt, F. Nori, and X. S. Ling, Phys. Rev. Lett. 74, 1206 (1995).

[6] D. S. Fisher, Phys. Rep. 301, 113 (1998).

[7] J. T. Uhl et al., Sci. Rep. 5, 16493 (2015).

[8] M.-Carmen Miguel, A. Vespignani, S. Zapperi, J. Weiss, and J. R. Grasso, Nature (London) 410, 667 (2001).

[9] D. Dimiduk, C. Woodward, R. LeSar, and M. D. Uchic, Science 312, 1188 (2006).

[10] N. Friedman, A. T. Jennings, G. Tsekenis, J.-Y. Kim, M. L. Tao, J. T. Uhl, J. R. Greer, and K. A. Dahmen, Phys. Rev. Lett. 109, 095507 (2012).

[11] M.-Carmen Miguel, A. Vespignani, M. Zaiser, and S. Zapperi, Phys. Rev. Lett. 89, 165501 (2002).

[12] J. Antonaglia, X. Xie, G. Schwarz, M. Wraith, J. W. Qiao, Y. Zhang, P. K. Liaw, J. T. Uhl, and K. A. Dahmen, Sci. Rep. 4, 4382 (2014).

[13] J. Antonaglia, W. J. Wright, X. J. Gu, R. R. Byer, T. C. Hufnagel, M. LeBlanc, J. T. Uhl, and K. A. Dahmen, Phys. Rev. Lett. 112, 155501 (2014).

[14] P. Moretti, M.-Carmen Miguel, M. Zaiser, and S. Zapperi, Phys. Rev. B 69, 214103 (2004).

[15] M.-Carmen Miguel, L. Laurson, and M. J. Alava, Eur. Phys. J. B. 64, 443 (2008).
[16] P. Y. Chan, G. Tsekenis, J. Dantzig, K. A. Dahmen, and N. Goldenfeld, Phys. Rev. Lett. 105, 015502 (2010).

[17] G. Tsekenis, N. Goldenfeld, and K. A. Dahmen, Phys. Rev. Lett. 106, 105501 (2011).

[18] K. A. Dahmen, Y. Ben-Zion, and J. T. Uhl, Phys. Rev. Lett. 102, 175501 (2009).

[19] K. A. Dahmen, Y. Ben-Zion, and J. T. Uhl, Nat. Phys. 7, 554 (2011).

[20] S. Song and T. Nieh, Intermetallics 17, 762 (2009).

[21] W. J. Wright, R. R. Byer, and X. J. Gu, Appl. Phys. Lett. 102, 241920 (2013).

[22] R. Maaß, P. M. Derlet, and J. R. Greer, Scr. Mater. 69, 586 (2013).

[23] W. J. Wright, Y. Liu, X. J. Gu, K. D. Van Ness, S. L. Robare, X. Liu, J. Antonaglia, M. LeBlanc, J. T. Uhl, T. C. Hufnagel, and K. A. Dahmen, J. Appl. Phys. 119, 084908 (2016).

[24] S. Papanikolaou, F. Bohn, R. Sommer, G. Durin, S. Zapperi, and J. P. Sethna, Nat. Phys. 7, 316 (2011).

[25] S. Papanikolaou, D. M. Dimiduk, W. Choi, J. P. Sethna, M. D. Uchic, C. F. Woodward, and S. Zapperi, Nature (London) 490, 517 (2012).

[26] D. S. Fisher, K. Dahmen, S. Ramanathan, and Y. Ben-Zion, Phys. Rev. Lett. 78, 4885 (1997).

[27] M. LeBlanc, L. Angheluta, K. Dahmen, and N. Goldenfeld, Phys. Rev. E 87, 022126 (2013).

[28] S. Ross, A First Course in Probability, 7th ed. (Pearson Prentice Hall, Upper Saddle River, NJ, 2006), p. 173.

[29] A. Dobrinevski, P. Le Doussal, and K. J. Wiese, Phys. Rev. E 85, 031105 (2012). 